\title{
Bionic Spider Robot Control Based on Image Processing Algorithm
}

\author{
Qingsheng Shi*, Cheng Wei and Ke Lu \\ College of Electrical Engineering, Henan University of Technology, Zhengzhou 450001, China \\ *Corresponding author
}

\begin{abstract}
Compared to wheeled and tracked robots, bionic robots are playing an increasingly important role in search, rescue, anti-terrorism and scientific investigation. The vision processing system is the vital part of the bionic robot system, and its algorithm determines the overall control performance of the system. In the paper, a bionic spider robot control strategy is proposed based on image processing algorithm. The object grasping of is taken as the control object, and the simulation experiment is carried out based on the self-made system. The experimental results show that the proposed control strategy based on image processing algorithm is feasible and effective.
\end{abstract}

Keywords- image processing; bionic spider robot; edge detection; object grasping

\section{INTRODUCTION}

Compared with wheeled and tracked mobile robots, walking robots have unique advantages in rugged terrain [1]. The emergence of bionic walking robot shows the advantages of walking robot. The walking trajectory of the robot is a series of discrete footprints, motion only discrete point of contact with the ground, the extent of damage to the environment is small, the rough terrain adaptability [2-3]. Therefore, bionic walking robots are more and more widely used in scientific exploration, scientific experiments, search, rescue and other conditions.

Bionic spider robot, as a typical bionic robot, through the nature of the spider in the biological movement of the body to simulate the completion of specific tasks [4-6]. As the core subsystem of humanoid spider robot, the vision system determines the performance of the system task. This paper studies the application of image processing algorithm in bionic spider robot object grasping operation. Using color camera to collect the surrounding environment photos, color enables the robot to distinguish the color, through the edge detection algorithm for image processing, which can screen the target contour features. After the image processing, the robot is able to obtain the position and orientation parameters of the target object as the judgment basis of the moving displacement, and then perform the grasping task.

\section{CONTROL STRATEGY OF BIONIC SPIDER ROBOT BASED ON IMAGE PROCESSING ALGORITHM}

Image processing is the core of bionic spider robot control strategy. The process mainly includes six steps: the first step: gray; the second step: Gauss filter; the third step: compute the gradient magnitude and direction; the fourth step: non maximu m suppression; the fifth step: selection of threshold; the sixth step:edge detection. Specific as follows:

The first step: grayscale [7]. This function is implemented by our custom function ImageProcess LoadImage (). First, read the pixel data from the camera module to handling from LCD GRAM, a total of $320 * 480=153600$ pixels, in this process will be in accordance with the RGB565 mode of the color pixels of 2 bytes split out three color components, mask definition RGB, $\mathrm{R}$ accounted for $5, \mathrm{G}$ accounted for $6, \mathrm{~B}$ accounted for 5 . According to the following formula, the 8 bit gray image is calculated:

$$
\text { Gray }=0.299 R+0.587 G+0.114 B
$$

Gray image conversion ImageProcess LoadImage (ImageResource* bmpImg) called void; after the function reads the pixel data from the LCD GRAM in the RGB565 mode according to the pixel resolution of 3 colors, as shown above the gray value according to the formula, the b mpImg para meter is the heap address stored gray data.

The second step: image Gauss filtering. The realization of image Gauss filtering can be realized by two times of one dimension Gauss kernel two times weighted, that is to say, first one-dimensional $\mathrm{X}$ direction convolution, and the result is further one-dimensional Y direction convolution.

The third step: to calculate the gradient and direction, the gradient of the gray value of the image is generally approximated by the first order finite difference, so that the two derivatives of the partial derivative of the image in the direction of $\mathrm{X}$ and $\mathrm{y}$ can be obtained. We use the commonly used Sobel operator [8]. For the image to be detected edge I, respectively in the horizontal $(\mathrm{x})$ direction and vertical direction (y) direction of differentiation, the method is the image I and convolution kernel $\mathrm{Gx}$ and $\mathrm{Gy}$ convolution, formula is expressed as follows:

$$
G_{x}=\left[\begin{array}{ccc}
-1 & 0 & 1 \\
-2 & 0 & 2 \\
-1 & 0 & 1
\end{array}\right] * I, G_{y}=\left[\begin{array}{ccc}
-1 & -2 & -1 \\
0 & 0 & 0 \\
1 & 2 & 1
\end{array}\right] * I
$$

After that, each point on the gradient image obtained in horizontal and vertical directions is executed:

$$
G=\sqrt{G_{x}^{2}+G_{y}^{2}}
$$


$G$ is the gradient image obtained by Sobel.

The fourth step: non maximum suppression. Non maxima suppression of amplitude image, can further eliminate non edge noise, more importantly, can refine the edge. The suppression logic is: along the gradient direction of the point, compare the amplitude of the previous two points, if the point is greater than the previous two points, then retain, if the point is less than the previous two points, then set to 0 .

The fifth step: double threshold selection. The mechanism of double threshold is first to specify a low threshold and a high threshold, high threshold for the whole image is generally the gray level distribution of the $70 \%$, and the high threshold for the low threshold of 1.5 to 2 times the size of; gray value greater than the high threshold, set to 255 , gray value is less than the low threshold, set to 0; gray value between high and low threshold, observed 8 pixels pixels near if there is a gray value of 255 , if not 255 , said this is a local maxima, isolated to be excluded, set to 0 ; if 255 , said this is a talk the other edge "border", set to 255 , then repeat the steps until the investigation after a pixel.

The sixth step: use the above interface function edge detection.

\section{EXPERIMENT AL SIMULATION AND ANALYSIS}

In order to verify the effectiveness of the proposed control strategy, based on the self-made system, the circular bottle cap was used as the grasping task, and the experimental verification was carried out under the laboratory conditions, as shown in figure 1 .

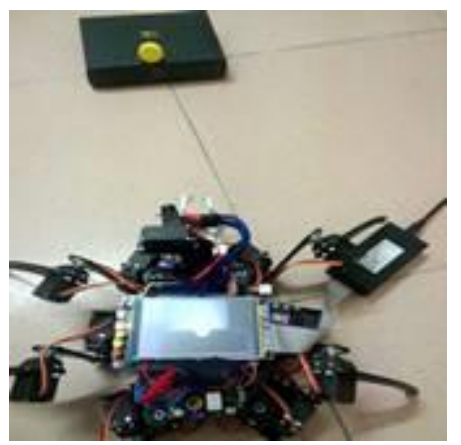

FIGURE I. IMAGE ACQUISITION

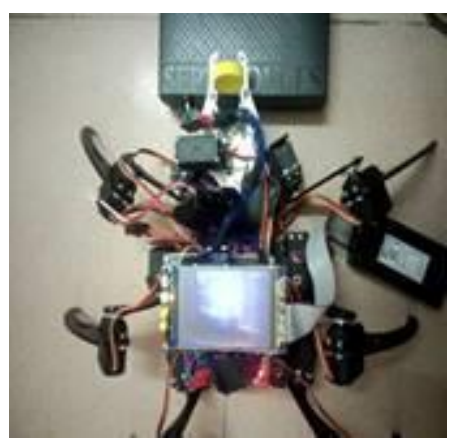

FIGURE II. ROBOT GRASPING RESULTS
It can be seen from Figure 2 that by using the designed control strategy, the spider robot can effectively capture the object by processing the image of the captured object.

\section{CONCLUSIONS}

The application of image processing algorithm in bionic spider robot control is studied in this paper. By gray, Gauss filter, gradient calculation, non maxima suppression, double threshold selection and edge detection operation, realizes the image processing of the bionic spider robot grasping object, and system steering image processing based on the results of objects quickly complete object action.

\section{ACKNOWLEDGMENT}

This work is supported by National Natural Science Foundation (NNCF) of China under Grant 61403124

\section{REFERENCES}

[1] D P Miller.Multiple behaviour-controlled micro-robots for planetary surface missions[C]. Proceedings of the IEEE International Conference on Systems,Man and Cybernetics.USA:IEEE, 1990:289-292.

[2] F Delcomyn,ME Nelson.Architectures for a biomimetic hexapod robot [J].Robotics and Aut onomous Systems, 2000,30(1):5-15.

[3] LIU Jing ZHAO Xiao-guang T AN Min.Legged Robots: A Review[J]. ROBOT, 2006,28(1):81-88.

[4] PENG Qian,LI Hongyan.design of Bionic and detectable spider robot system[J].research and exploration in laboratory,2016,35(12):84-87.

[5] M Ghayour, A Zareei. Inverse Kinematic Analysis of a Hexapod Spider-Like Mobile Robot[J].Advanced Materials Research, 2011, 403 408: 5061-5067.

[6] SHI Wenzao, WANG Ping.Desgin and implementation of bionic spider robot [J].Electronic Sci.\&Tech.2013,26(3):90-92.

[7] Rafael C. Gonzalez, Richard E. Woods.Digital Image Processing (Third Edition), Publishing House oof Electronics Industry, 2011.

[8] XU Yanlei,ZHAO Jiyin,JIAO Yubin.Study of edge detection method for noisy image[J].Application research of computers, 2009,26(1): 387-389. 